Published in final edited form as:

Schizophr Res. 2012 September ; 140(1-3): 87-92. doi:10.1016/j.schres.2012.06.012.

\title{
Affect Recognition in People at Clinical High Risk of Psychosis
}

\author{
Jean Addington ${ }^{\mathrm{a},}{ }^{*}$, Danijela Piskulic ${ }^{\mathrm{a}}$, Diana Perkins ${ }^{\mathrm{b}}$, Scott W. Woods ${ }^{\mathrm{c}}$, Lu Liu ${ }^{\mathrm{a}}$, and David \\ L. Penn ${ }^{d}$ \\ aDepartment of Psychiatry, Faculty of Medicine, University of Calgary, Calgary AB, Canada \\ ${ }^{b}$ Department of Psychiatry, University of North Carolina at Chapel Hill, Chapel Hill NC, United \\ States \\ 'Department of Psychiatry, Yale University, New Haven, CT, USA \\ dDepartment of Psychology, University of North Carolina at Chapel Hill, Chapel Hill NC, United \\ States
}

\begin{abstract}
Individuals with schizophrenia demonstrate stable deficits in affect recognition. Similar deficits in affect recognition have been observed in those who are at clinical high risk (CHR) of developing psychosis. The current project aimed to longitudinally examine affect processing in CHR individuals, to determine if affect processing predicted later conversion to psychosis and if affect processing deficits were unique to those who met established criteria for prodromal syndromes. The sample consisted of 172 CHR and 100 help-seeking individuals (HS) who were followed for up to 24 months. All CHR individuals met the Criteria of Prodromal States (COPS) based on the Structured Interview for Prodromal Symptoms (SIPS). The SIPS was used to determine conversion to psychosis. Affect recognition was assessed using two facial affect recognition tasks and a measure of affective prosody. In comparison to previously published data from nonpsychiatric controls, both CHR and HS groups demonstrated deficits on affect recognition. By 2 years 25 CHR participants converted to psychosis. Interestingly, there were no differences between converters and non-converters on any affect recognition tasks. This is one of the first studies to longitudinally examine affect processing and its relationship to later conversion to psychosis in individuals at-risk for psychosis. While poorer affect recognition may be associated with vulnerability for psychosis, the current results suggest that it may not be a marker of developing a psychotic illness.
\end{abstract}

\footnotetext{
(C) 2012 Elsevier B.V. All rights reserved.

*Corresponding author. Department of Psychiatry, 3280 Hospital Drive NW, Calgary AB T2N 4Z6, Canada. Tel: +1 (403) 2016379 , Fax: + 1 (403) 2109182.

Publisher's Disclaimer: This is a PDF file of an unedited manuscript that has been accepted for publication. As a service to our customers we are providing this early version of the manuscript. The manuscript will undergo copyediting, typesetting, and review of the resulting proof before it is published in its final citable form. Please note that during the production process errors may be discovered which could affect the content, and all legal disclaimers that apply to the journal pertain.

Conflict of interest

There are no conflicts of interest for any of the authors with respect to the data in this paper or for the study.

Contributors

Drs Addington, Perkins and Woods were responsible for the design of the whole study and the supervision of all aspects of data collection. Dr Penn contributed to the design and data collection with respect to the affect processing data. Ms Liu and Dr Piskulic were responsible for data analysis. Dr Addington took the lead on writing the manuscript with help in writing from Piskulic, Woods, $\&$ Perkins. All authors contributed to and approved the final manuscript.
} 


\section{Keywords}

psychosis prodrome; social cognition; affect recognition; facial affect; affective prosody

\section{Introduction}

There is a growing interest in social cognition in schizophrenia, mainly due to its association with poor social functioning (Fett et al., 2011). One of the most studied domains of social cognition is affect recognition. It has been well established that individuals with schizophrenia demonstrate stable deficits in both discrimination and identification of affect irrespective of modality, facial (Addington et al., 2006a, Horan et al., in press; Pinkham et al., 2007) or prosodic (Edwards et al., 2001; Kucharska-Pietura et al., 2005), and across all stages of illness (Green et al., in press). Similar deficits in affect recognition have been observed in individuals who are putatively prodromal for psychosis, i.e., at clinical high risk (CHR) of developing a psychotic disorder. CHR individuals, relative to healthy controls, have demonstrated impaired performance on facial affect identification comparable to the performance of individuals with a first episode of psychosis and those who have a more chronic course of psychosis (Addington et al., 2008; Green et al., 2011). Amminger et al (Amminger et al., in press), using both a facial affect task as well as a measure of affective prosody, reported similar results relative to healthy controls and first episode patients; CHR individuals exhibited deficits in the recognition of fear and sadness across both face and voice modalities compared to non-psychiatric controls.

Although these studies suggest that affect recognition deficits may be a traitcharacteristic, there are no longitudinal studies testing the stability of affect recognition in those at clinical high risk or examining its relationship to conversion to psychosis. The aim of this project was to examine longitudinally affect processing in a large sample of individuals at CHR of psychosis, to determine if affect processing was a predictor of later conversion to psychosis and to determine if deficits in affect processing were unique to those who met established criteria for a prodromal syndrome.

\section{Method}

\subsection{Sample}

The overall sample consisted of 172 individuals (98 males, 74 females) at CHR of psychosis with a mean age of $19.8(\mathrm{SD}=4.5)$ years and 100 help-seeking individuals, the help-seeking controls (HSC) (56 males, 44 females) with a mean age of $19.4(\mathrm{SD}=3.9)$ years. All were participants in the PREDICT study that was conducted at the Universities of Toronto (70 CHR, 45 HSC), North Carolina (62 CHR, 31 HSC) and Yale (40 CHR, 24 HSC). PREDICT was designed to determine predictors of conversion to psychosis. All CHR individuals met the Criteria of Prodromal States (COPS) based on the Structured Interview for Prodromal Symptoms (SIPS) (McGlashan et al., 2010). Participants were excluded if they were using antipsychotics at baseline. Furthermore, antipsychotics were not used at any later points in this study. One hundred and sixty-eight CHR participants met attenuated positive symptom syndrome (APSS) criteria, which include the emergence or worsening of a non-psychotic level disturbance in thought content, thought process or perceptual abnormality over the past year. Four participants met criteria for genetic risk and deterioration (GRD), which required either a first degree relative with a psychotic disorder or the subject having schizotypal personality disorder (SPD) plus at least a $30 \%$ drop in functioning on the General Assessment of Functioning (GAF) scale in the past 12 months. The HSC group were individuals who had (i) responded to recruitment efforts for the CHR sample, and (ii) on a phone screen appeared likely to meet prodromal criteria but after the initial comprehensive 
interview did not. The HSC group consisted of the following groups: (i) family high risk but no decline in functioning $(\mathrm{n}=17)$, (ii) long standing symptoms i.e. attenuated positive symptoms had been present for more than one year $(n=47)$, current prodromal symptoms but symptoms were clearly due to another disorder $(n=2)$, (iii) only had negative symptoms $(n=4)$ and (iv) the remaining group reported vague symptoms that neither met severity nor frequency $(n=30)$. Those with longstanding symptoms were individuals who had attenuated psychotic symptoms that had begun or worsened more than a year before and were rated 3-5 on severity on the Scale of Prodromal Symptoms (SOPS). This severity is within the prodromal range, but to meet prodromal criteria attenuated symptoms have to have begun or worsened in the past year.

The Structured Clinical Interview for DSM-IV (SCID-I) (First et al., 1995) was used to determine the presence of any axis I disorders. Participants were excluded if they met criteria for any current or lifetime axis I psychotic disorder, prior history of treatment with an antipsychotic, or past or current history of a clinically significant central nervous system disorder which may confound or contribute to clinical high risk symptoms. A comprehensive clinical assessment was conducted by the PI or clinical psychiatrist or psychologist at each site to determine if entry criteria were met. Only 146 of the CHR participants and 85 of the HSC completed the affect recognition tasks. In addition, since this longitudinal study lasted for four years, the first person recruited could have had four years of follow-up whereas the last person may have only had 3 months. Therefore at each followup, missing subjects are accounted for either by conversion to psychosis, missing the assessment, dropping out of the study or not being in the study long enough to reach that particular follow-up. See Table 1.

\subsection{Measures}

Criteria for a prodromal syndrome and criteria for conversion to psychosis were determined using the Structured Interview for Prodromal Syndromes (SIPS) (McGlashan et al., 2010).

Conversion meant that at least one of the five attenuated positive symptoms reached a psychotic level of intensity (rated 6) for a frequency of $\geq 1$ hour/day for 4 days/week during the past month or that symptoms seriously impacting functioning (e.g. severely disorganised or dangerous to self or others) (McGlashan et al., 2010). Symptoms were assessed with the Scale of Prodromal Symptoms (SOPS), which consists of 19 items in 4 symptom domains: positive, negative, general and disorganized. Intelligence was assessed using the Block design, Arithmetic, Digit Symbol/Coding, Vocabulary and Information subtests from the Wechsler Adult Intelligence Test (WAIS)/Wechsler Intelligence Scale for Children-III (WISC-III) (Wechsler, 1974; 1981).

Facial affect recognition was assessed with the Facial Emotion Identification Test (FEIT) and the Facial Emotion Discrimination Test (FEDT) (Kerr and Neale, 1993). These measures have been described in detail elsewhere (Addington et al., 2008). Affective prosody (AP) was assessed using a task developed by Edwards and colleagues (Edwards et al., 2001) and used in the Amminger study (Amminger et al., in press). The task involves audio recordings of 4 simple sentences (i.e. "he will come soon", "they must stay here", "she will drive fast" and "we must go there") spoken by three professional actors displaying following emotions: fear, sadness, anger, surprise and neutral resulting in a total of 60 items. Based on sentence recordings participants were required to indicate which emotion was expressed. For each actor, there were 3 practice and 20 target items, with eight seconds of silence between each item. Published reliability coefficients (Chronbach's alpha) for AP is 0.85 (Edwards et al., 2001), for FEDT is 0.68, (Pinkham and Penn, 2006) and FEIT is 0.50 (Pinkham and Penn, 2006). 


\subsection{Procedures}

This was a longitudinal study of predictors of conversion to psychosis whereby all three sites recruited CHR and HS individuals. Raters were experienced research clinicians who demonstrated adequate reliability at routine reliability checks. Gold standard post-training agreement on the distinction between high risk and psychotic levels of intensity on the positive symptom items (i.e., the critical threshold for determining initial eligibility and subsequent conversion status) was excellent (kappa $=0.90)$. The DSM-IV diagnoses were made using the SCID-I. Interrater reliability was determined at the start of the study and annually by $100 \%$ agreement on the diagnosis and at least $80 \%$ agreement for symptom presence. JA chaired weekly conference calls to review criteria for all individuals admitted to the study. Affect processing assessments were conducted by trained research assistants trained by DLP. The study protocols and informed consents were reviewed and approved by the ethical review boards of all three study sites.

\subsection{Statistical Analyses}

All analyses were performed with the use of IBM SPSS version 19 and SAS version 9.2. The Student t-test and chi-square test were used to compare baseline differences between the CHR group and the HS group and between the converters and the non-converters. Mann Whitney U test was used to compare converters and non-converters given the unequal sample sizes in the two groups. Spearman correlations were used to determine associations amongst measures. To accommodate missing data and account for intra-participant correlation over time, generalized linear mixed model for repeated measures was used to examine changes over time (baseline, 6 months, 12 months, 18 months and 24 months) and group differences for ratings on the three affect recognition measures.

\section{Results}

\subsection{Sample Characteristics}

There were no demographic differences between the CHR and HSC groups. Within the CHR group there were no differences between those who converted and those who did not convert within the time of the study. These results are presented in Table 2 . The CHR group had significantly higher ratings on attenuated positive symptoms and on general symptoms on the SOPS compared to the HSC. These results are presented in Table 3. The groups were also compared on IQ. There were no group differences (CHR, M=111.65, SD=17.13; HSC, $\mathrm{M}=110.75, \mathrm{SD}=19.99, \mathrm{t}=-0.32)$.

Since there were a number of dropouts in this study, we compared those who dropped out on demographics, IQ, three affect recognition tasks and symptoms. There were no differences at any time between HSC who completed assessments and those who did not complete. For the CHR participants who dropped out before one year those who dropped out had significantly higher scores on the facial identification task $(t=2.06 \mathrm{p}<0.05)$. For those who completed one year but not two years $(n=8)$ compared to those who completed beyond one year those who dropped out after one year had lower scores on facial affect discrimination $(\mathrm{t}=3.56, \mathrm{p}<0.01)$, less negative symptoms $(\mathrm{t}=2.65, \mathrm{p}<0.1)$.

\subsection{Affect recognition}

In the HSC group, all three affect recognition tasks were significantly associated with one another (Pearson's correlation coefficient $r$ ranged from 0.31-0.35, all $p<0.001$ ). In the CHR group, the facial affect identification and discrimination tasks were associated $(r=0.2$, $\mathrm{p}<0.05$ ) and the facial affect identification task was significantly related to the prosody task $(\mathrm{r}=0.36, \mathrm{p}<0.001)$. There were some associations amongst the affect recognition tasks and symptoms. Interestingly, none of the tasks were associated with positive symptoms in either 
group. See Table 4. Table 5 presents the means and SDs for each affect task for each group. In this table we have also presented means from healthy control groups published in the literature (Addington et al., 2006; Edwards et al., 2001; Kee et al., 2004; Mueser et al., 1996; Pinkham and Penn, 2006; Salem et el., 1996).

Results of the mixed effects modelling demonstrated that there were no differences between the CHR and the HSC group on affect recognition tasks at baseline or any of the follow up assessments. However, for the HSC group, there was a significant improvement on all affect tasks by 24 months. For the CHR group, improvement occurred on two of the tasks by 6 months and on one task again at 24 months. However, since there is a significant loss of data at 18 and 24 months we tested these results running the mixed effects model up to 12 months only. The only change was that for the CHR group there was a significant change in the FEIT from baseline to 6 months $(\mathrm{p}=0.22)$ where as previously this had not been significant $(\mathrm{p}=0.057)$. These results are presented in Table 6 .

At baseline we examined the differences between the CHR and HSC groups on the individual emotions within the prosody task and there were no significant differences.

Within the first 2 years of the study 25 participants in the CHR group, 13 males and 12 females, converted to psychosis. There were no differences between those who converted and those who did not convert to psychosis on any of the three affect recognition tasks at baseline. For those who converted there were no changes over time on any of the affect recognition tasks.

\section{Discussion}

This is one of the first studies to longitudinally examine affect processing and its relationship to later conversion to psychosis in a group of people at high risk of developing psychosis. In this study, we did not have a healthy control group, thus we cannot definitely say that our CHR sample demonstrated affect recognition deficits relative to healthy controls. However, an earlier publication (Addington et al., 2008) demonstrated that a subsample of $85 \mathrm{CHR}$ individuals had significant deficits relative to healthy controls on the facial affect identification task and performed similarly to those with an established psychotic illness. With respect to the prosody task, our samples had scores that fell in the same range as those of clinical samples with schizophrenia and other psychoses in Edwards et al's study (Edwards et al., 2001). We have reported results from other studies which suggest that our sample of individuals at CHR of psychosis most likely have deficits in affect processing with respect to facial affect identification and affective prosody. Interestingly, the performance of the HSC group was indistinguishable from those at CHR. There was no difference in performance on the three measures at baseline between those who did and did not develop psychosis. Those at CHR showed some improvement at 6 months and for those who remained in the study at 24 months there was some evidence of improved performance.

There are some limitations to this study. The follow-up sample is small due to the time line of the study. The number of conversions is approximately $17 \%$ by 2 years which is an average of what has been reported in the literature (Yung et al., 2008) and since all participants did not reach the one year mark, there may be more potential converters. Our assessment of social cognition was limited to affect processing and it may be that an assessment of other domains of social cognition would have had different results. However, with respect to psychotic disorders, affect processing is one of the best studied and robust of the domains of social cognition (Kohler et al., 2010; Hoekert et al., 2007). We did not observe any differences between the groups on individual emotions nor did any of the 
individual emotions predict conversion to psychosis. The strengths of the current study are its longitudinal, prospective design, large initial sample, examination of two modalities of affect processing as a predictor to later conversion to psychosis and the fact that the sample was free of antipsychotics.

Thus, our results suggest that poorer performance on social cognition may be indicative of being potentially vulnerable to developing psychosis but not necessarily a marker of developing a full blown psychotic illness. This fits with the suggestion that there is a continuum of psychosis from psychotic like-experiences to subclinical psychotic symptoms to psychosis (van Os et al., 2009). Thus, difficulties in processing affect information may be a problem for those who report psychotic-like-experiences or fluctuating subclinical psychotic symptoms, or subthreshold symptoms regardless of whether they meet criteria for a putative prodrome experience. In fact a recent internet-based, general population study demonstrated that individual differences in psychosis-proneness were associated with the ability to process facial affect (Germine and Hooker, 2011). Germine et al., (Germine et al., 2011) further demonstrated that in individuals who rated high on social anhedonia, there was less neural activity in brain regions thought to be implicated in facial emotion processing. In addition there is evidence of deficits in affect recognition in those who are at family high risk (Kee et al, 2004).

However, we cannot entirely rule out the predictive role of affect processing for conversion to psychosis. The number of converters was small and approximately half of the conversions occurred in the first 6 months of the study. Of those who converted after 6 months and had at least one follow-up pre-conversion, statistically, there was no significant change in their scores over time. Furthermore, a visual examination of their scores revealed that a few had declining scores and a few had scores that varied only by one or two points over time. This is the difficulty when focusing only on average performance.

Thus, it is likely that these individuals at high risk of psychosis, regardless of their level of vulnerability (i.e., meeting criteria for a syndrome), have persistent difficulties with affect processing. For those who do not go on to develop a psychotic illness, there seems to be some improvement over time, but for those who do develop a psychotic illness the difficulties may persist. Since the field of social cognition needs further work, particularly with respect to norms of the many measures that are used, we are unable to determine the level of improvement that may be occurring.

Our results may have important implications for social functioning since the ability to accurately recognize emotion is fundamental to social functioning. Difficulties in affect recognition as well as other aspects of social cognition are consistently associated with poor social functioning (Fett et al., 2011) and have been reported to be the best predictor of social functioning in schizophrenia even when compared with other cognitive tasks (Hooker and Park, 2002). A number of studies of individuals at CHR have reported deficits in functioning (Addington et al. 2008; Cornblatt et al., in press) and that social deficits may be predictive of conversion to later psychosis (Cornblatt et al., in press). Thus, although poor affect processing may exist in CHR samples and does not independently predict conversion, it does relate to functioning, which in turn has been reported to predict conversion.

\section{Acknowledgments}

Role of funding source

Funding for this study was provided by NIMH grants U01MH06634-02 to J.Addington, U01MH066069-04 to D. Perkins, and U01MH066160 to S.Woods. The NIMH had no further role in study design; in the collection, analysis and interpretation of data; in the writing of the report; and in the decision to submit the paper for publication. 


\section{References}

Addington J, Penn D, Woods SW, Addington D, Perkins DO. Facial affect recognition in individuals at clinical high risk for psychosis. Br. J. Psychiatry. 2008; 192:67-68. [PubMed: 18174514]

Addington J, Saeedi H, Addington D. Facial affect recognition: a mediator between cognitive and social functioning in psychosis? Schizophr. Res. 2006; 85:142-150. [PubMed: 16678388]

Amminger GP, Schafer MR, PapageorgiouU K, Klier CM, Schlogelhofer M, Mossaheb N, WerneckRohrer S, Nelson B, McGorry PD. Emotion Recognition in Individuals at Clinical High-Risk for Schizophrenia. Schizophr. Bull. in press.

Cornblatt BA, Carrion RE, Addington J, Seidman L, Walker EF, Cannon TD, Cadenhead KS, McGlashan TH, Perkins DO, Tsuang MT, Woods SW, Heinssen R, Lencz T. Risk Factors for Psychosis: Impaired Social and Role Functioning. Schizophr. Bull. in press.

Edwards J, Pattison PE, Jackson HJ, Wales RJ. Facial affect and affective prosody recognition in first episode schizophrenia. Schizophr. Res. 2001; 48:235. [PubMed: 11295377]

Fett AK, Viechtbauer W, Dominguez MD, Penn DL, van OS J, Krabbendam L. The relationship between neurocognition and social cognition with functional outcomes in schizophrenia: a metaanalysis. Neurosci. Biobehav. Rev. 2011; 35:573-588. [PubMed: 20620163]

First, M.; Spitzer, R.; Gibbon, M.; Williams, B.; Williams, J. Structured Clinical Interview for DSMIV Axis I Disorders. Patient Edition. New York: New York State Psychiatric Institute; 1995.

Germine LT, Hooker CI. Face emotion recognition is related to individual differences in psychosisproneness. Psychol. Med. 2011; 41:937-947. [PubMed: 20810004]

Germine LT, Garrido L, Bruce L, Hooker C. Social anhedonia is associated with neural abnormalities during face emotion processing. Neuroimage. 2011; 58:935-945. [PubMed: 21763435]

Green MF, Bearden CE, Cannon TD, Fiske AP, Hellemann GS, Horan WP, Kee K, Kern RS, Lee J, Sergi MJ, Subotnik KL, Sugar CA, Ventura J, Yee CM, Nuechterlein KH. Social Cognition in Schizophrenia, Part 1: Performance Across Phase of Illness. Schizophr. Bull. in press.

Hoekert M, Kahn RS, Pijnenborg M, Aleman A. Impaired recognition and expression of emotional prosody in schizophrenia: Review and meta-analysis. Schizophr. Res. 2007; 96:135. [PubMed: 17766089]

Hooker C, Park S. Emotion processing and its relationship to social functioning in schizophrenia patients. Psychiatry Res. 2002; 112(1):41-50. [PubMed: 12379449]

Horan WP, Green MF, DeGroot M, Fiske A, Hellemann G, Kee K, Kern RS, Lee J, Sergi MJ, Subotnik KL, Sugar CA, Ventura J, Nuechterlein KH. Social Cognition in Schizophrenia, Part 2: 12-Month Stability and Prediction of Functional Outcome in First-Episode Patients. Schizophr. Bull. in press.

Kee K, Horan W, Mintz J, Green M. Do the siblings of schizophrenia patients demonstrate affect perception deficits? Schizoph Bull. 2004; 67:87.

Kerr SL, Neale JM. Emotion perception in schizophrenia: specific deficit or further evidence of generalized poor performance? J. Abnorm. Psychol. 1993; 102:312-318. [PubMed: 8315144]

Kohler CG, Walker JB, Martin EA, Healey KM, Moberg PJ. Facial emotion perception in schizophrenia: A meta-analytic review. Schizophr. Bull. 2010; 36(5):109-119. [PubMed: 19959707]

Kucharska-Pietura K, David AS, Masiak M, Phillips ML. Perception of facial and vocal affect by people with schizophrenia in early and late stages of illness. Br. J. Psychiatry. 2005; 187:523. [PubMed: 16319404]

McGlashan, T.; Walsh, B.; Woods, S. The Psychosis-Risk Syndrome: Handbook for Diagnosis and Follow-up. New York: Oxford University Press; 2010.

Mueser KT, Doonan R, Penn DL, Blanchard JJ, Bellack AS, Nishith P, DeLeon J. Emotion recognition and social competence in chronic schizophrenia. J. Abnorm. Psychol. 1996; 105:271. [PubMed: 8723008]

Pinkham AE, Penn DL. Neurocognitive and social cognitive predictors of interpersonal skill in schizophrenia. Psych. Res. 2006; 143(2-3):167. 
Pinkham AE, Penn D, Perkins DO, Graham K, Siegel M. Emotion perception and social skill over the course of psychosis: a comparison of individuals at risk, and early and chronic schizophrenia spectrum illness. Cog. Neuropsychiatry. 2007; 12:198.

Salem JE, Kring AM, Kerr SL. More evidence for generalised poor performance in facial emotion perception in schizophrenia. J.Abnorm.Psychol. 1996; 105:480. [PubMed: 8772021]

van Os J, Linscott RJ, Myin-Germeys I, Delespaul P, Krabbendam L. A systematic review and metaanalysis of the psychosis continuum: evidence for a psychosis proneness-persistence-impairment model of psychotic disorder. Psychol. Med. 2009; 39:179-195. [PubMed: 18606047]

Yung AR, Nelson B, Stanford C, Simmons MB, Cosgrave EM, Killackey E, Phillips LJ, Bechdolf A, Buckby J, McGorry PD. Validation of "prodromal" criteria to detect individuals at ultra high risk of psychosis: 2 year follow-up. Schizophr. Res. 2008; 105:10-17. [PubMed: 18765167] 


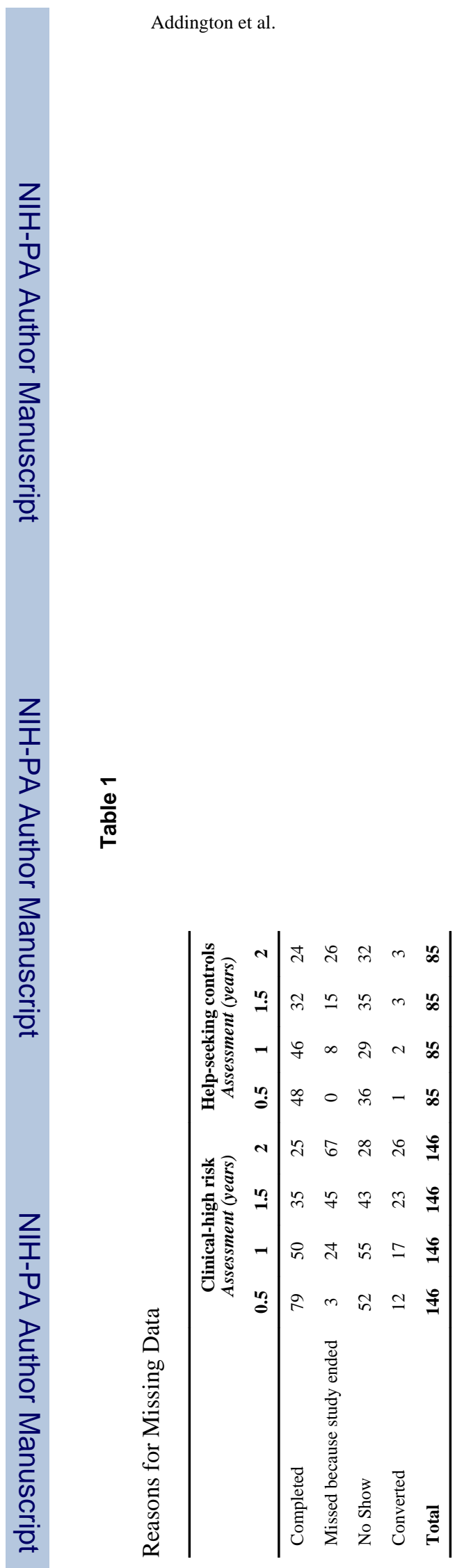

Schizophr Res. Author manuscript; available in PMC 2013 September 01. 


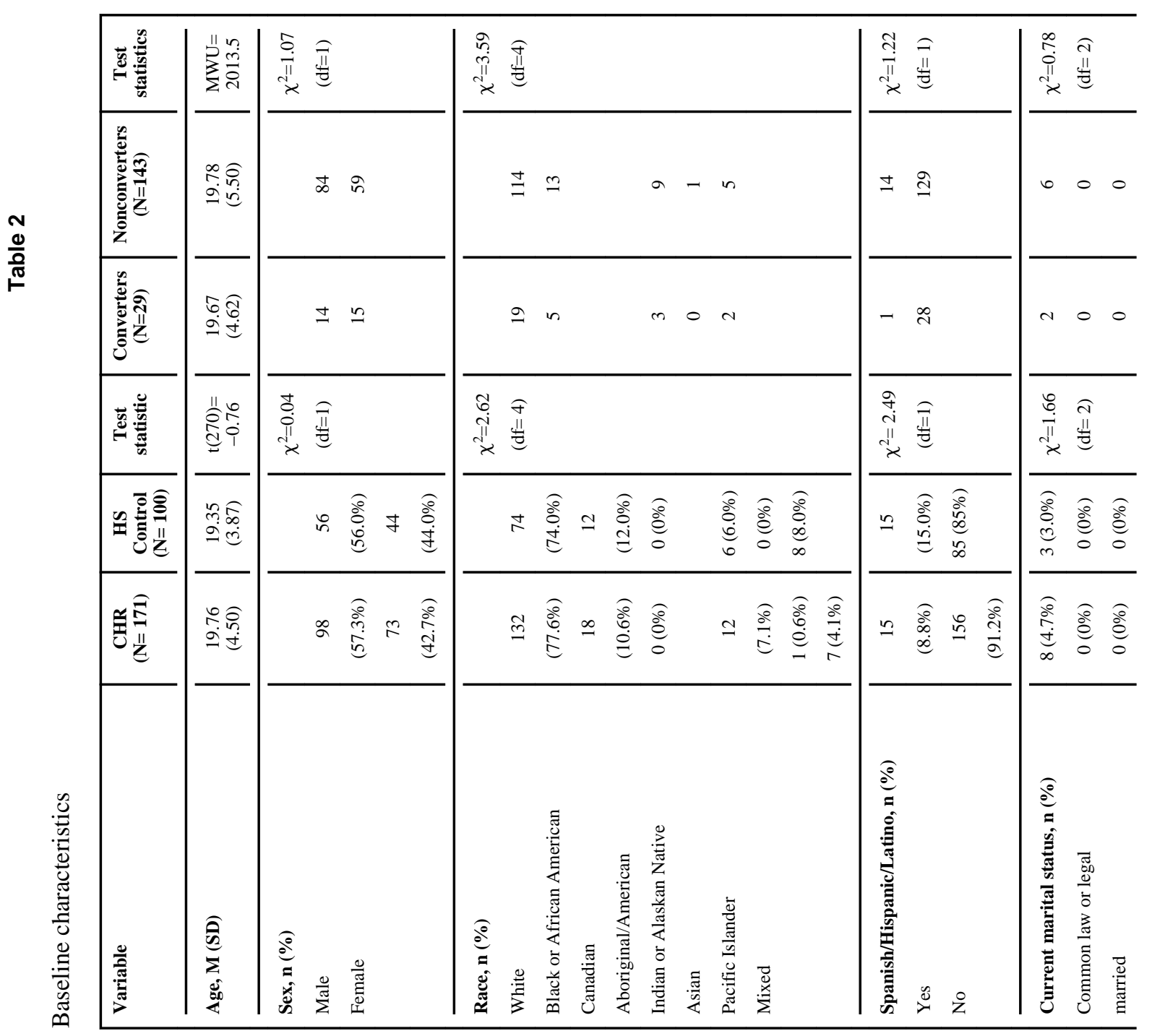




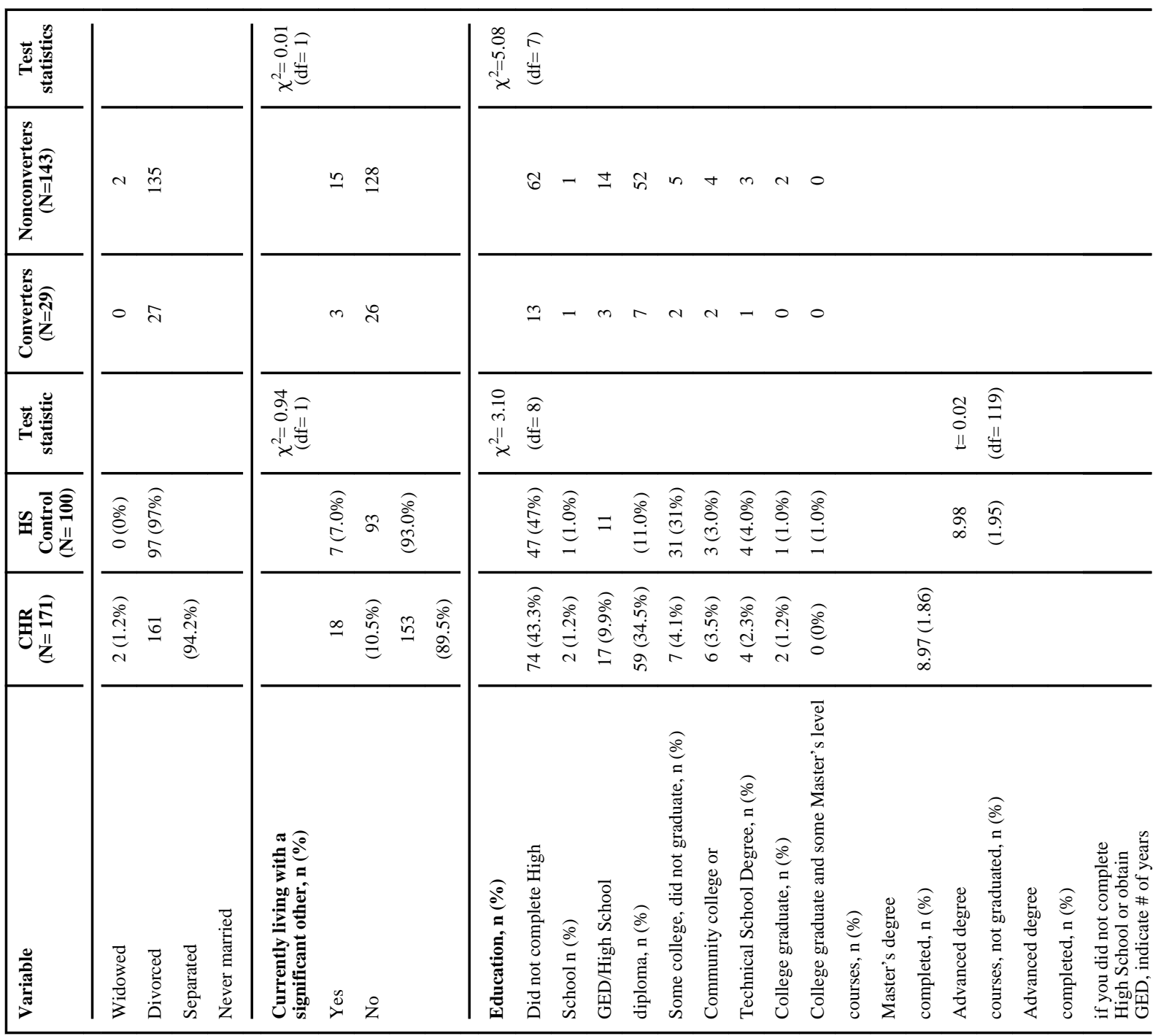




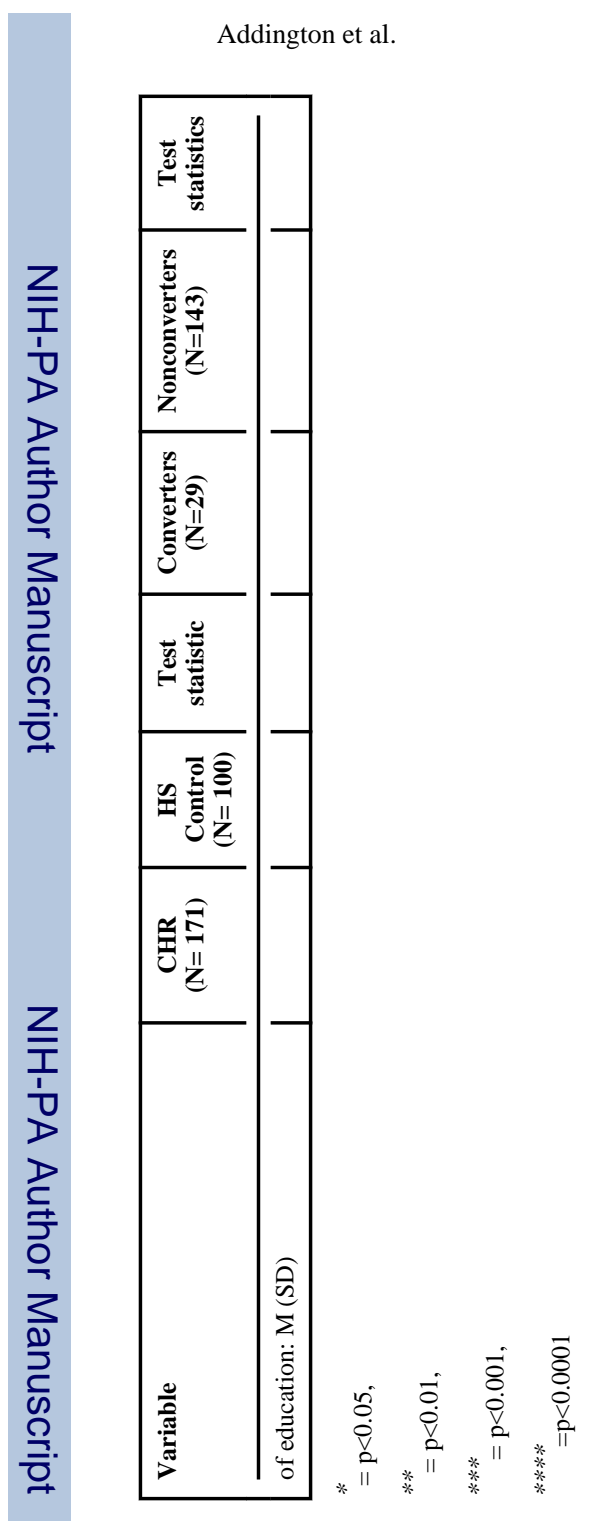

Page 12

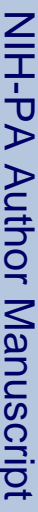

Schizophr Res. Author manuscript; available in PMC 2013 September 01. 
Table 3

SOPS Symptom Ratings for Clinical High Risk and Help Seeking Control Groups

\begin{tabular}{lccl}
\hline $\begin{array}{c}\text { Symptom } \\
\text { subscores }\end{array}$ & $\begin{array}{c}\text { CHR } \\
\text { (N= 172) } \\
\text { M (SD) }\end{array}$ & $\begin{array}{c}\text { HSC } \\
\text { (N= 100) } \\
\mathbf{M} \text { (SD) }\end{array}$ & t- value \\
\hline Positive & $11.02(3.20)$ & $6.53(4.15)$ & $-9.31^{* * *}$ \\
Negative & $8.61(5.66)$ & $8.38(6.30)$ & -0.30 \\
Disorganised & $4.11(2.75)$ & $3.68(3.16)$ & -1.18 \\
General & $7.02(3.91)$ & $5.19(4.11)$ & $-3.64 * * *$ \\
\hline$* * *$ & & & \\
p $\leq 0.001$ & & & \\
\hline
\end{tabular}




\section{Table 4}

Correlations between SOPS Symptoms and Affect Recognition at Baseline

\begin{tabular}{ccccc}
\hline \multicolumn{5}{c}{ Clinical High Risk } \\
& Positive & Negative & Disorganised & General \\
\hline FEDT & 0.11 & 0.02 & -0.03 & -0.01 \\
FEIT & -0.01 & $-0.27^{* *}$ & -0.17 & -0.14 \\
AP & -0.09 & $-0.22^{* *}$ & -0.12 & -0.01 \\
\hline \multicolumn{5}{c}{ Help-seeking Controls } \\
\hline FEDT & -0.13 & -0.07 & -0.13 & -0.07 \\
FEIT & -0.03 & -0.11 & -0.03 & -0.02 \\
AP & -0.08 & $-0.38^{* *}$ & $-0.33^{* *}$ & 0.01 \\
\hline
\end{tabular}

Note: all correlations are Spearman's rho coefficients;

*** p 0.007 following Bonferroni correction FEIT=Facial Affect Identification Test; FEDT= Facial Affect Discrimination Test; AP= Affect Prosody 


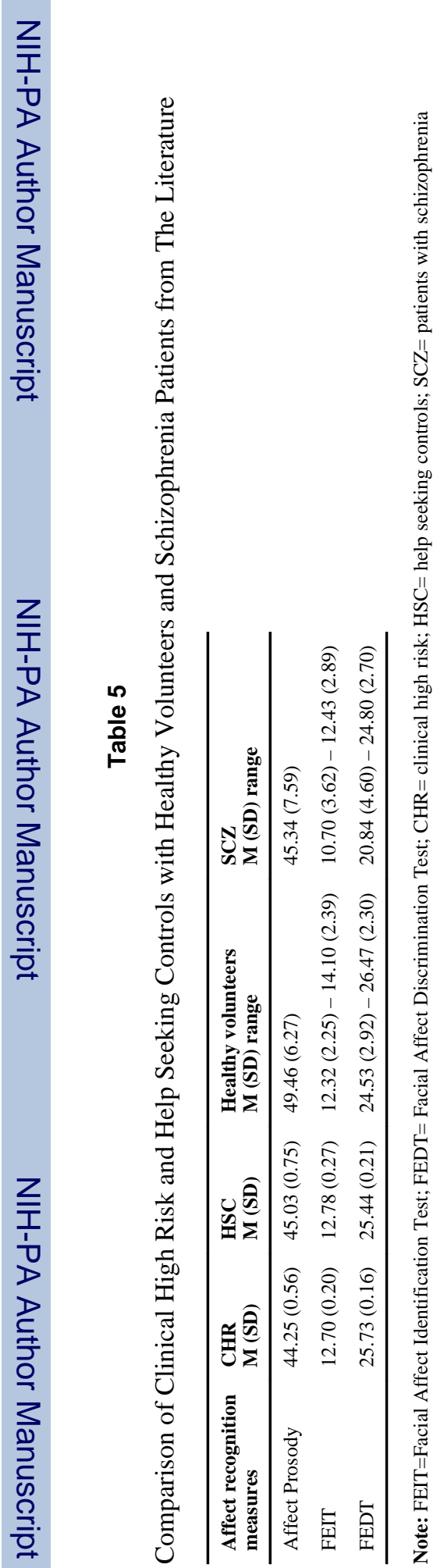

Schizophr Res. Author manuscript; available in PMC 2013 September 01. 
